This item was submitted to Loughborough's Research Repository by the author.

Items in Figshare are protected by copyright, with all rights reserved, unless otherwise indicated.

\title{
Optimization of intelligent reflecting surface assisted full-duplex relay networks
}

PLEASE CITE THE PUBLISHED VERSION

https://doi.org/10.1109/LWC.2020.3031343

PUBLISHER

IEEE

VERSION

AM (Accepted Manuscript)

\section{PUBLISHER STATEMENT}

(c) 2020 IEEE. Personal use of this material is permitted. Permission from IEEE must be obtained for all other uses, in any current or future media, including reprinting/republishing this material for advertising or promotional purposes, creating new collective works, for resale or redistribution to servers or lists, or reuse of any copyrighted component of this work in other works.

\section{LICENCE}

\section{All Rights Reserved}

\section{REPOSITORY RECORD}

Abdullah, Zaid, Gaojie Chen, Sangarapillai Lambotharan, and Jonathon A. Chambers. 2020. "Optimization of Intelligent Reflecting Surface Assisted Full-duplex Relay Networks”. Loughborough University. https://hdl.handle.net/2134/19213470.v1. 


\title{
Optimization of Intelligent Reflecting Surface Assisted Full-Duplex Relay Networks
}

\author{
Zaid Abdullah, Member, IEEE, Gaojie Chen, Senior Member, IEEE, Sangarapillai Lambotharan, Senior \\ Member, IEEE, and Jonathon A. Chambers, Fellow, IEEE
}

\begin{abstract}
In this work, we propose a novel hybrid communication network that utilizes both a Full-Duplex (FD) Decodeand-Forward (DF) relay and an Intelligent Reflecting Surface (IRS) to support data transmission over wireless channels. We design the reflecting coefficients at the IRS to maximize the minimum achievable rate of the two hops for the proposed hybrid network. To that end, we utilize a change-of-variables with SemiDefinite Relaxation (SDR) approach to overcome the non-concave objective function and the non-convex optimization constraints. Our results demonstrate that the proposed hybrid IRS with FD relay scheme is able to achieve a significant performance gain over both the hybrid IRS with Half-Duplex (HD) relay as well as the IRS-only scheme, given that the self-interference at the relay is sufficiently suppressed.
\end{abstract}

Index Terms-Intelligent Reflecting Surface, Full-Duplex, Max-Min Optimization

\section{INTRODUCTION}

$\mathbf{I}$ $\mathrm{N}$ the last two decades, numerous technologies have been proposed to meet the exponentially increasing demand of data traffic over wireless channels. One of these technologies is the state-of-the-art Intelligent Reflecting Surfaces (IRSs), which have rapidly gained a massive interest among researchers and are expected to have a key role in future wireless networks. A typical IRS array consists of a planar surface with a large number of passive reflecting elements that can be programmed to control the propagation environment in a constructive manner [1], [2]. As IRS arrays do not require power amplifiers or radio frequency chains to reflect signals with certain amplitudes and/or phase-shifts [3], they can provide cost-effective and energy-efficient communication links [4], [5].

In principle, IRS technology is similar to the well-known Amplify-and-Forward (AF) relaying, and the main difference between the two technologies is that classical relaying requires power amplifiers to amplify signals before being forwarded to the destination. There have been many recent studies that have focused on comparing the performance of the two different schemes. For example, the authors in [6] compared the two technologies in terms of the achievable rates, and their results demonstrated that hundreds of reflecting elements at the IRS

This work was supported by the Engineering and Physical Sciences Research Council under Grants EP/R006377/1 and EP/R006385/1.

Z. Abdullah, G. Chen and J. A. Chambers are with the School of Engineering, University of Leicester, Leicester, LE1 7RU, UK. Emails: \{zaid.abdullah; gaojie.chen; jonathon.chambers\} @ leicester.ac.uk.

S. Lambotharan is with the Wolfson School of Mechanical, Electrical and Manufacturing Engineering, Loughborough University, Loughborough LE11 3TU, U.K. Email: s.lambotharan@lboro.ac.uk. are required to outperform a multi-antenna AF relay. In [7], the authors showed that although a multi-antenna AF relay can achieve higher data rates than the IRS in low and moderate Signal-to-Noise Ratios (SNRs), the latter has higher Energy Efficiency (EE) as it does not require active power amplifiers, and thus has a much lower power consumption than classical relaying.

In contrast, the authors in [8] demonstrated that for an IRS to outperform a single-antenna Half-Duplex (HD) Decodeand-Forward (DF) relay, hundreds of reflecting elements are required at the IRS, and it is only at very high data rates that an IRS would be more energy-efficient than the HD-DF relay. Moreover, Full-Duplex (FD) DF relaying was compared to IRS in [9], where the authors showed that sufficiently large IRSs are required to outperform an FD-DF relay. More recently, in our previous work in [10], we proposed a hybrid HD-DF relay and IRS system, and our work demonstrated that in practical scenarios, utilizing a single-antenna HD-DF relay can provide a significant performance enhancement for an IRS supported system, and at no additional power cost; while in [11], the authors proposed a relay-aided IRS network, where two IRSs are connected via an FD relay with directional transmit and receive antennas.

In this work, we propose a novel hybrid FD relay and IRS assisted network, where an FD-DF relay and an IRS array jointly support the communication between a source and a destination node. Note that in our previous work in [10], an HD relay was adopted for the hybrid IRS-assisted network, and despite the significant gain obtained in low and medium SNRs, at high SNRs, the limited bandwidth of the HD relay becomes the main bottleneck which highly affects the overall performance of such hybrid systems. Therefore, we aim to overcome this disadvantage by adopting an FD relay that is capable of utilizing the full bandwidth for simultaneous transmission and reception. Specifically, a max-min optimization problem is formulated for the design of the phase-shifts at the IRS and for the allocation of transmit powers at the source and the FD relay to maximize the minimum rate of the two hops, yielding a highly improved performance at medium and high SNR regimes compared to the hybrid system proposed in [10]. Moreover, unlike the work in [11] which requires two efficiently isolated IRSs to enable FD transmission, here we demonstrate that with the proposed phase-shifts design, a single IRS array can be amalgamated with an FD relay, and the proposed scheme shows a significant performance gain compared to a system which only comprises an FD-relay or an IRS to support data transmission. In addition, the work in 
[11] requires an FD relay with two horn (directional) antennas dedicated to the two IRSs (i.e. the relay only receives/transmits signals from/to the two IRSs); while in our work the FD relay is equipped with omnidirectional antennas and can receive/transmit signals from/to all nodes in the network. This gives our proposed scheme more flexibility since the relay can be at any location in the cell and does not have to be dedicated to the IRS.

Our main contributions can be summarized as follows:

- We propose a novel IRS assisted network with FDDF relay, where both the IRS and the relay are efficiently utilized to support the transmission between a source/destination pair.

- The solution to the resultant optimization problem for the phase-shifts design is nontrivial, hence we propose a change-of-variables with Semi-Definite Relaxation (SDR) approach to tackle the non-concave objective function and non-convex optimization constraints. Moreover, we utilize the obtained phase-shifts at the IRS and perform optimal power allocation to further enhance the performance of the network.

- Our results demonstrate that the proposed hybrid IRS with FD relay significantly outperforms the hybrid IRS with HD relay and the case where only an IRS is utilized to support data transmission.

Notations: Matrices and vectors are represented by boldface uppercase and lowercase letters, respectively. $\operatorname{Tr}(\mathbf{A})$ is the trace of matrix $\mathbf{A}$, and $\mathbf{A} \succeq 0$ indicates that matrix $\mathbf{A}$ is positive semi-definite. $|x|$ and $\mathbb{E}\{x\}$ are the absolute and expected values of $x$, respectively. $\mathbf{a}^{T}$ and $\mathbf{a}^{H}$ are the transpose and Hermitian transpose of a, respectively. $[\mathbf{A}]_{m, n}$ denotes the $n$th element of the $m$ th row of $\mathbf{A}$, and $\mathbf{a}_{i}$ is the $i$ th element of a. $\operatorname{diag}\{\mathbf{a}\}$ is a diagonal matrix whose diagonals are the elements of a. Finally, $\arg (x)$ denotes the phase of a complex number $x$.

\section{SySTEM MODEL}

We consider a system that comprises a Source (S), Destination (D), FD-DF relay (R), and IRS (I) array with $N$ reflecting elements as depicted in Fig. 1. We assume that $\mathrm{S}$ and $\mathrm{D}$ are far apart from each other and there is no direct link between them. Let $\mathbf{h}_{\mathrm{SI}}^{H} \in \mathbb{C}^{1 \times N}, \mathbf{h}_{\mathrm{ID}} \in \mathbb{C}^{N \times 1}, \mathbf{h}_{\mathrm{IR}} \in \mathbb{C}^{N \times 1}, \mathbf{h}_{\mathrm{RI}}^{H} \in \mathbb{C}^{1 \times N}$, $h_{\mathrm{SR}} \in \mathbb{C}, h_{\mathrm{RD}} \in \mathbb{C}$, and $h_{\mathrm{RR}} \in \mathbb{C}$ be the links from $\mathrm{S} \rightarrow \mathrm{I}$, $\mathrm{I} \rightarrow \mathrm{D}, \mathrm{I} \rightarrow \mathrm{R}, \mathrm{R} \rightarrow \mathrm{I}, \mathrm{S} \rightarrow \mathrm{R}, \mathrm{R} \rightarrow \mathrm{D}$, and the selfinterference link or $\mathrm{R}$, respectively. Apart from $h_{\mathrm{RR}}$, all links are assumed to experience both small and large-scale fading, and can be expressed as $\mathbf{h}_{j}=\mathbf{g}_{j} d_{j}^{-\alpha / 2}, j \in\{\mathrm{SI}, \mathrm{ID}, \mathrm{RI}, \mathrm{IR}\}$, and $h_{k}=g_{k} d_{k}^{-\alpha / 2}, k \in\{\mathrm{SR}, \mathrm{RD}\}$, where $g \in \mathbb{C}$ and the elements of $\mathrm{g} \in \mathbb{C}^{N}$ are the small-scale Rayleigh fading channels with zero mean and unit variance, $d_{j}$ is the distance between two nodes in the $j$ th link, and $\alpha$ is the path-loss exponent. We assume block fading channels, where channel coefficients remain constant for the duration of transmitting one block of data, and change independently from one block to another. Moreover, let $\boldsymbol{\Theta}=\operatorname{diag}\left\{e^{j \boldsymbol{\theta}_{1}}, \ldots . ., e^{j \boldsymbol{\theta}_{N}}\right\}$ be a diagonal matrix that controls the phase-shifts for each element

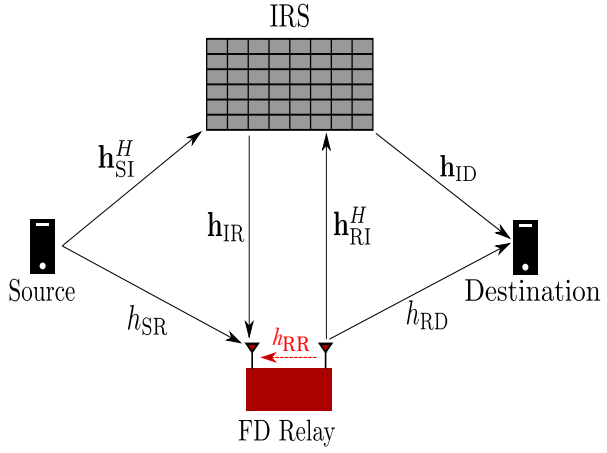

Fig. 1: The proposed system model with a source, a relay, an IRS, and a destination.

at the IRS array ${ }^{1}$. At time instant $t_{m}$, the source transmits the $m$ th symbol of the $l$ th block $x_{l}(m)$ to $\mathrm{R}$, and at the same time, $\mathrm{R}$ transmits the $m$ th symbol of the previous block $x_{l-1}(m)$ to D. Therefore, and assuming that powers of signals reflected by the IRS more than once can be neglected, the received signal at $\mathrm{R}$ can be given as follows

$$
\begin{aligned}
y_{\mathrm{R}}\left(t_{m}\right) & =\sqrt{P_{1}}\left(\mathbf{h}_{\mathrm{SI}}^{H} \boldsymbol{\Theta} \mathbf{h}_{\mathrm{IR}}+h_{\mathrm{SR}}\right) x_{l}(m) \\
& +\sqrt{P_{2}}\left(\mathbf{h}_{\mathrm{RI}}^{H} \boldsymbol{\Theta} \mathbf{h}_{\mathrm{IR}}+h_{\mathrm{RR}}\right) x_{l-1}(m)+n_{\mathrm{R}}\left(t_{m}\right),
\end{aligned}
$$

where $P_{1}$ and $P_{2}$ are the transmit powers of $\mathrm{S}$ and $\mathrm{R}$, respectively, $n_{R}$ is the additive white Gaussian noise (AWGN) at R with zero mean and variance of $\sigma^{2}$. Accordingly, and assuming that $\mathbb{E}\left\{\left|x_{l}(m)\right|^{2}\right\}=1,(m \in\{1, \ldots, M\}, l \in\{1, \ldots, L\})$, the received Signal-to-Interference and Noise Ratio (SINR) at R can be expressed as follows

$$
\gamma_{1}=\frac{P_{1}\left|\mathbf{h}_{\mathrm{SI}}^{H} \boldsymbol{\Theta} \mathbf{h}_{\mathrm{IR}}+h_{\mathrm{SR}}\right|^{2}}{\gamma_{\mathrm{rr}}+\sigma^{2}} .
$$

where $\gamma_{\mathrm{rr}}$ is the residual self-interference power after performing self-interference cancellation ${ }^{2}$. In contrast, the received signal at $\mathrm{D}$ can be given as follows

$$
\begin{aligned}
y_{\mathrm{D}}\left(t_{m}\right) & =\sqrt{P_{2}}\left(\mathbf{h}_{\mathrm{RI}}^{H} \boldsymbol{\Theta} \mathbf{h}_{\mathrm{ID}}+h_{\mathrm{RD}}\right) x_{l-1}(m) \\
& +\sqrt{P_{1}}\left(\mathbf{h}_{\mathrm{SI}}^{H} \boldsymbol{\Theta} \mathbf{h}_{\mathrm{ID}}\right) x_{l}(m)+n_{\mathrm{D}}\left(t_{m}\right),
\end{aligned}
$$

where $n_{\mathrm{D}}$ is the AWGN at D with zero mean and variance of $\sigma^{2}$. Note that in this case $x_{l}(m)$ is the interference signal for $\mathrm{D}$, while $x_{l-1}(m)$ represents the signal of interest. Therefore, the SINR at D can be expressed as

$$
\gamma_{2}=\frac{P_{2}\left|\mathbf{h}_{\mathrm{RI}}^{H} \Theta \mathbf{h}_{\mathrm{ID}}+h_{\mathrm{RD}}\right|^{2}}{P_{1}\left|\mathbf{h}_{\mathrm{SI}}^{H} \Theta \mathbf{h}_{\mathrm{ID}}\right|^{2}+\sigma^{2}} .
$$

${ }^{1} \mathrm{We}$ assume that all reflecting elements have reflecting amplitudes of 1 , and perfect CSI is available for the phase-shifts design. Note that these assumptions might not hold exactly in reality, and here we try to demonstrate the performance limits of such hybrid schemes assuming an ideal scenario.

${ }^{2}$ In addition to passive cancellation via antenna separation, absorptive shielding, and cross-polarization, active cancellation can be performed in the digital domain as follows. The self-interference channel $h_{r r}=$ $\sqrt{P_{2}}\left(\mathbf{h}_{\mathrm{RI}}^{H} \Theta \mathbf{h}_{\mathrm{IR}}+h_{\mathrm{RR}}\right)$ can be estimated at $\mathrm{R}$ after receiving each block by computing the correlation between the received signal $y_{\mathrm{R}}\left(t_{m}\right)$ and the transmitted signal $x_{l-1}(m)$. Considering the $m$ th symbol, we have $\mathbb{E}\left\{y_{\mathrm{R}}\left(t_{m}\right) x_{l-1}^{*}(m)\right\}=\sqrt{P_{1}}\left(\mathbf{h}_{\mathrm{SI}}^{H} \boldsymbol{\Theta} \mathbf{h}_{\mathrm{IR}}+h_{\mathrm{SR}}\right) \mathbb{E}\left\{x_{l}(m) x_{l-1}^{*}(m)\right\}+$ $\sqrt{P_{2}}\left(\mathbf{h}_{\mathrm{RI}}^{H} \Theta \mathbf{h}_{\mathrm{IR}}+h_{\mathrm{RR}}\right) \mathbb{E}\left\{x_{l-1}(m) x_{l-1}^{*}(m)\right\}+\mathbb{E}\left\{n_{\mathrm{R}}\left(t_{m}\right) x_{l-1}^{*}(m)\right\}=$ $\hat{h}_{r r}$, since $\mathbb{E}\left\{x_{l}(m) x_{l-1}^{*}(m)\right\}=\mathbb{E}\left\{n_{\mathrm{R}}\left(t_{m}\right) x_{l-1}^{*}(m)\right\}=0$, while $\mathbb{E}\left\{x_{l-1}(m) x_{l-1}^{*}(m)\right\}=1$. 
Therefore, and given the fact that $\mathrm{R}$ follows a DF protocol, the achievable rate for the proposed hybrid scheme can be expressed as

$$
\begin{aligned}
\mathcal{R} & =\min \left\{\mathcal{R}_{1}, \mathcal{R}_{2}\right\} \\
& =\min \left\{\log _{2}\left(1+\gamma_{1}\right), \log _{2}\left(1+\gamma_{2}\right)\right\},
\end{aligned}
$$

where $\mathcal{R}_{i}$ is the achievable rate for the $i$ th hop, $i \in\{1,2\}$. Accordingly, our goal is to maximize the minimum rate between the two hops by designing the phase-shifts at I, and carrying out an Optimal Power Allocation (OPA) between S and $\mathrm{R}$ under a total power constraint, as will be thoroughly explained in the next section.

\section{MAX-Min OPTIMIZATION}

For a two-hop system with DF relaying, the maximum rate is achieved when both hops have the same SINRs, or equivalently when $\mathcal{R}_{1}=\mathcal{R}_{2}$. Therefore, the corresponding max-min optimization problem can be expressed as follows

$$
\begin{aligned}
& \underset{\Theta, P_{1}, P_{2}}{\operatorname{maximize}} \min _{i \in\{1,2\}} \mathcal{R}_{i} \\
& \text { subject to } \\
& \left|[\Theta]_{n, n}\right|=1, \forall n \in\{1, \ldots, N\}, \\
& P_{1}+P_{2} \leq P_{T},
\end{aligned}
$$

where $P_{T}$ is the total available power for the system. Problem (6) has a non-concave objective function with a non-convex unit-modulus constraint. Moreover, the optimization parameters are coupled as can be seen in (2) and (4), which make the optimization difficult to handle. Accordingly, we divide problem (6) into two sub-problems. In particular, we start by optimizing the phase-shifts assuming Equal Power Allocation (EPA) between $\mathrm{S}$ and $\mathrm{R}$. Then, once the optimal phase-shifts are obtained, we shift our attention to the optimal split of the total available power as will be explained in detail in the following subsections. Note that carrying out alternating optimization for the phase-shifts and transmit powers can lead to a better performance in terms of the achievable rates, however, that would be at the cost of increased computational complexity.

\section{A. Optimizing the phase-shifts with EPA}

Let us start by defining $\boldsymbol{\theta}^{H}=\left[e^{j \boldsymbol{\theta}_{1}}, \ldots, e^{j \boldsymbol{\theta}_{N}}\right], \mathbf{v}^{H}=$ $\left[\boldsymbol{\theta}^{H}, 1\right], \boldsymbol{\beta}_{\mathrm{SIR}}=\operatorname{diag}\left\{\mathbf{h}_{\mathrm{SI}}^{H}\right\} \mathbf{h}_{\mathrm{IR}}, \boldsymbol{\beta}_{\mathrm{RID}}=\operatorname{diag}\left\{\mathbf{h}_{\mathrm{RI}}^{H}\right\} \mathbf{h}_{\mathrm{ID}}, \boldsymbol{\beta}_{\mathrm{SID}}=$ $\operatorname{diag}\left\{\mathbf{h}_{\mathrm{SI}}^{H}\right\} \mathbf{h}_{\mathrm{ID}}, \mathbf{f}_{s}=\left[\begin{array}{c}\boldsymbol{\beta}_{\mathrm{SIR}} \\ h_{\mathrm{SR}}\end{array}\right], \mathbf{q}_{s}=\left[\begin{array}{c}\boldsymbol{\beta}_{\mathrm{SID}} \\ 0\end{array}\right]$, and $\mathbf{q}_{r}=\left[\begin{array}{c}\boldsymbol{\beta}_{\mathrm{RID}} \\ h_{\mathrm{RD}}\end{array}\right]$. Then, an equivalent representation of the achievable rates for the first and second hops can be expressed as

$$
\begin{aligned}
& \mathcal{R}_{1}=\log _{2}\left(1+\frac{P_{1}\left|\mathbf{v}^{H} \mathbf{f}_{s}\right|^{2}}{\gamma_{\mathrm{rr}}+\sigma^{2}}\right), \\
& \mathcal{R}_{2}=\log _{2}\left(1+\frac{P_{2}\left|\mathbf{v}^{H} \mathbf{q}_{r}\right|^{2}}{P_{1}\left|\mathbf{v}^{H} \mathbf{q}_{s}\right|^{2}+\sigma^{2}}\right) .
\end{aligned}
$$

Maximizing the minimum of $\mathcal{R}_{1}$ and $\mathcal{R}_{2}$ in their current forms will still result in a non-concave objective function with nonconvex constraints. However, now we can apply change-ofvariables with SDR to overcome the aforementioned problems. By letting $P_{1}=P_{2}=P, \mathbf{F}_{S}=P \mathbf{f}_{s} \mathbf{f}_{s}^{H}, \mathbf{Q}_{S}=P \mathbf{q}_{s} \mathbf{q}_{s}^{H}, \mathbf{Q}_{R}=$ $P \mathbf{q}_{r} \mathbf{q}_{r}^{H}, \mathbf{Q}=\mathbf{Q}_{S}+\mathbf{Q}_{R}, \mathbf{V}=\mathbf{v} \mathbf{v}^{H}$, and after following the

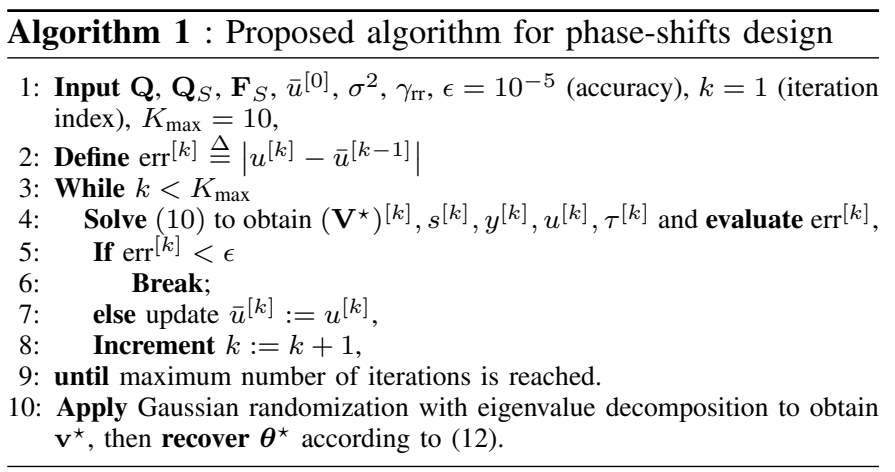

change-of-variables approach in [12], we can now introduce the following exponential slack variables

$$
\begin{aligned}
e^{s} & =\operatorname{Tr}\left(\mathbf{F}_{S} \mathbf{V}\right)+\gamma_{\mathrm{rr}}+\sigma^{2}, \\
e^{y} & =\operatorname{Tr}(\mathbf{Q V})+\sigma^{2}, \\
e^{u} & =\operatorname{Tr}\left(\mathbf{Q}_{S} \mathbf{V}\right)+\sigma^{2} .
\end{aligned}
$$

Next, after following simple logarithmic and exponential properties and dropping the rank-1 constraint on $\mathbf{V}$ through SDR, and introducing an additional slack variable $\tau$, the optimization problem for the phase-shifts design can be expressed as follows $^{3}$

$$
\begin{aligned}
& \underset{\mathbf{V}, s, y, u, \tau}{\operatorname{maximize}} \quad \tau \\
& \text { subject to } \\
& s-\ln \left(\gamma_{\mathrm{rr}}+\sigma^{2}\right) \geq \tau, \\
& y-u \geq \tau, \\
& \operatorname{Tr}(\mathbf{F} S \mathbf{V})+\gamma_{\mathrm{rr}}+\sigma^{2} \geq e^{s}, \\
& \operatorname{Tr}(\mathbf{Q V})+\sigma^{2} \geq e^{y}, \\
& \operatorname{Tr}\left(\mathbf{Q}_{S} \mathbf{V}\right)+\sigma^{2} \leq e^{u}, \\
& {[\mathbf{V}]_{n, n}=1, \forall n \in\{1, \ldots, N+1\},} \\
& \mathbf{V} \succeq 0,
\end{aligned}
$$

the only non-convex constraint in (9) is (9e) and needs to be linearized. Accordingly, a first order Taylor approximation is applied such that $e^{u}=e^{\bar{u}}(u-\bar{u}+1)$, where the linearization is made around $\bar{u}$. Therefore, (9) is recast as

$$
\begin{aligned}
& \underset{\mathbf{V}, s, y, u, \tau}{\operatorname{maximize}} \quad \tau \\
& \text { subject to } \\
& \operatorname{Tr}\left(\mathbf{Q}_{S} \mathbf{V}\right)+\sigma^{2} \leq e^{\bar{u}}(u-\bar{u}+1), \\
& \text { and constraints (9a), (9b), (9c), (9d), (9f) and (9g). }
\end{aligned}
$$

At this point, all constraints are convex, and problem (10) can be solved iteratively using software packages such as CVX. It should be noted that before the iterative algorithm begins, $\bar{u}$ is initialized as follows

$$
\bar{u}^{[0]}=\ln \left(\operatorname{Tr}\left(\mathbf{Q}_{S} \tilde{\mathbf{V}}\right)+\sigma^{2}\right),
$$

where $\tilde{\mathbf{V}}$ is any feasible solution. Moreover, once a convergence or maximum number of optimization iterations is reached, the optimal solution $\mathbf{v}^{\star}$ can be obtained via eigenvalue decomposition if the rank of $\mathbf{V}$ is 1 . Otherwise, Gaussian randomization with eigenvalue decomposition is applied to

${ }^{3}$ It is worth to point out that $(\ln 2)^{-1}$ is omitted from the objective function without loss of optimality. 


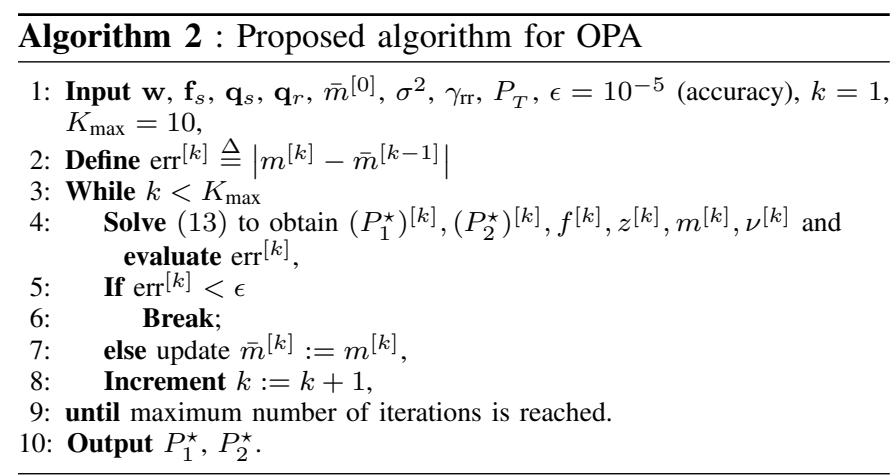

approximate $\mathbf{v}^{\star}$ as explained in detail in [13]. Then, the optimized phase-shifts $\boldsymbol{\theta}^{\star}$ can be recovered from $\mathbf{v}^{\star}$ as follows

$$
\boldsymbol{\theta}_{n}^{\star}=\exp \left(j \arg \left(\frac{\mathbf{v}_{n}^{\star}}{\mathbf{v}_{N+1}^{\star}}\right)\right), \forall n \in\{1, \ldots, N\} .
$$

It should be noted that such SDR schemes followed by a sufficiently large number of randomizations guarantee a minimum accuracy of $\pi / 4$ of the optimal objective value [6], [14]. The steps of the proposed optimization scheme are explained in detail in Algorithm 1.

\section{B. Max-Min power allocation}

Once the phase-shifts at the IRS are optimized, we focus our attention on optimizing the distribution of the total available power for further rate enhancement. Let us define $\mathbf{w}^{H}=$ $\left[\left(\boldsymbol{\theta}^{\star}\right)^{H}, 1\right]$; recalling the formulations of $\mathcal{R}_{1}$ and $\mathcal{R}_{2}$ in (7a) and (7b), and after following a similar approach (change-ofvariables and Taylor approximation), we obtain the following optimization problem

$$
\begin{aligned}
& \underset{\substack{P_{1}, P_{2}, f, z, m, \nu}}{\operatorname{maximize}} \quad \nu \\
& \text { subject to } \\
& f-\ln \left(\gamma_{\mathrm{rr}}+\sigma^{2}\right) \geq \nu, \\
& z-m \geq \nu \text {, } \\
& P_{1}\left|\mathbf{w}^{H} \mathbf{f}_{s}\right|^{2}+\gamma_{\mathrm{rr}}+\sigma^{2} \geq e^{f}, \\
& P_{1}\left|\mathbf{w}^{H} \mathbf{q}_{s}\right|^{2}+P_{2}\left|\mathbf{w}^{H} \mathbf{q}_{r}\right|^{2}+\sigma^{2} \geq e^{z}, \\
& P_{1}\left|\mathbf{w}^{H} \mathbf{q}_{s}\right|^{2}+\sigma^{2} \leq e^{\bar{m}}(m-\bar{m}+1), \\
& P_{1}+P_{2} \leq P_{T} \text {. }
\end{aligned}
$$

where $f, z, m, \nu$ are slack variables. Problem (13) and its constraints are convex and can be efficiently solved using CVX. Moreover, the initial value of $\bar{m}$ is set based on the EPA criterion as follows

$$
\bar{m}^{[0]}=\ln \left(\frac{1}{2} P_{T}\left|\mathbf{w}^{H} \mathbf{q}_{s}\right|^{2}+\sigma^{2}\right),
$$

The steps of the proposed OPA scheme are shown in detail in Algorithm 2.

\section{RESULTS AND DISCUSSION}

Unless stated otherwise, the source was located at the origin of a $2 \mathrm{D}$ plane, i.e. $\left(x_{\mathrm{S}}, y_{\mathrm{S}}\right)=(0,0)$, while $\left(x_{\mathrm{D}}, y_{\mathrm{D}}\right)=(60,0)$, $\left(x_{\mathrm{I}}, y_{\mathrm{I}}\right)=(30,5)$, and $\left(x_{\mathrm{R}}, y_{\mathrm{R}}\right)=(30,-5)$ (all in meters). $\gamma_{\mathrm{rr}}$ was assumed to be $0 \mathrm{dBW}$, the path-loss exponent $\alpha=2.5$, and $\sigma^{2}=1$. We compare our proposed hybrid IRS with FD

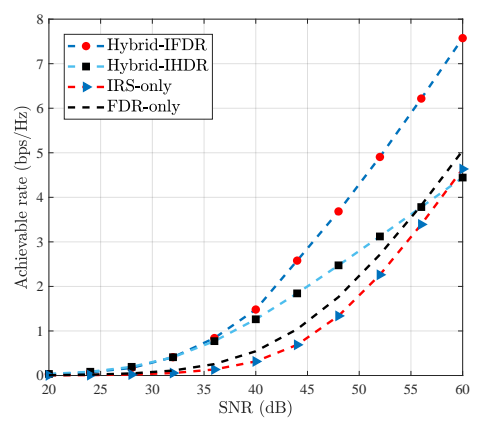

Fig. 2: Achievable rate vs SNR (dB) for different schemes when $N=32$.

Relay (Hybrid-IFDR) with three different schemes: the hybrid IRS with HD Relay (Hybrid-IHDR) in [10], the IRS-only scheme, and the FD Relaying (FDR) only scheme. Moreover, to ensure fair comparisons, we optimize the power allocation of the Hybrid-IHDR and FDR schemes to maximize the achievable rates under a total power constraint of $P_{1}+P_{2}=$ $P_{T}$ Watts, while for the IRS only scheme, the source transmits with the total available power of $P_{T}$ Watts. Finally, the SNR is defined as $P_{T} / \sigma^{2}$.

Fig. 2 demonstrates the achievable rate performance for a wide range of SNRs. The proposed Hybrid-IFDR scheme significantly outperforms all other relaying schemes, especially at moderate and high SNR values. For example, to achieve a rate of $4 \mathrm{bps} / \mathrm{Hz}$, the proposed Hybrid-IFDR scheme outperforms the Hybrid-IHDR, IRS-only, and FDR-only schemes by 8.3, 9, and $7.6 \mathrm{~dB}$ of transmit SNRs. Moreover, as the SNR increases, the performance gap between the Hybrid-IFDR and the IRSonly scheme remains almost the same, meaning that even at high SNRs, the proposed hybrid-IFDR can provide significant performance gain compared to an IRS-only assisted network, which is not the case for the Hybrid-IHDR.

In Fig. 3, the performance of the considered relaying schemes is investigated for a wide range of values of $N$, i.e. number of reflecting elements at the IRS. The proposed hybrid-IFDR scheme significantly outperforms the IRS-only approach even when the number of elements is large and the SNR is high. For example, when the IRS is equipped with 64 reflecting elements, the performance of an IRS-only assisted system shows almost the same performance as the hybrid-IHDR approach, while the hybrid-IFDR scheme shows a significant gain of $2.2 \mathrm{bps} / \mathrm{Hz}$ compared to a system that only utilizes an IRS to support data transmission, given that the SNR is $50 \mathrm{~dB}$.

Moreover, the performance of different relaying protocols is investigated in Fig. 4 as a function of the location of R. Similar to the hybrid-IHDR, the optimal performance for the proposed hybrid-IFDR scheme is achieved when $\mathrm{R}$ is closest to the IRS. Nevertheless, our results demonstrate the advantages of such hybrid schemes even when the relay is far away from the IRS. For example, in the worst case scenario, i.e. when $x_{R}=50$ meters, a gain of $1.23 \mathrm{bps} / \mathrm{Hz}$ is still obtained for our proposed system compared to that which only utilizes an IRS 


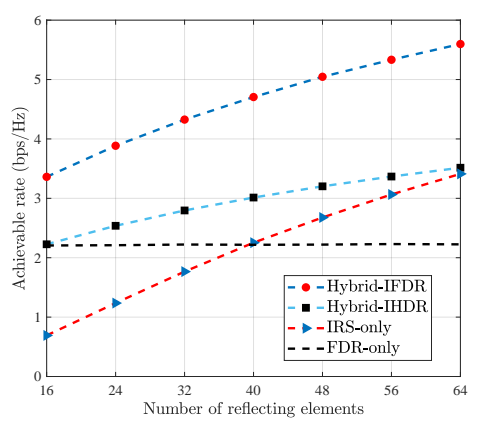

Fig. 3: Achievable rate vs number of reflecting elements at the IRS $(N)$ when the $\mathrm{SNR}=50 \mathrm{~dB}$.

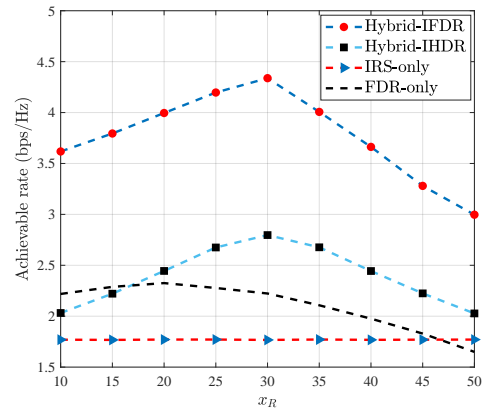

Fig. 4: Achievable rate for different locations of $\mathrm{R}$ when $N=$ 32 and $\mathrm{SNR}=50 \mathrm{~dB}$.

to support data transmission. This demonstrates the flexibility of such hybrid schemes, as even if the relay was far away from the IRS, it can still provide a considerable performance gain. In other words, the relay does not have to be implemented specifically to assist the IRS, however, the closer the relay is to the IRS, the higher gains that can be obtained.

Fig. 5 highlights the importance of the self-interference cancellation accuracy to guarantee the advantages of such hybrid schemes. In particular, the hybrid-IFDR outperforms the hybrid-IHDR approach as long as the power of the residual self-interference is less than $8.5 \mathrm{dBW}$. In addition, the proposed hybrid-IFDR outperforms an IRS-only supported system for all values of $\gamma_{\mathrm{rr}}$ less than $14 \mathrm{dBW}$. Finally, from all the presented results, the proposed hybrid-IFDR scheme is always better than that which only considers an FD relay to enhance link quality. This demonstrates that the performance of an FD relay can always be enhanced when combined with an IRS, as long as the phase-shifts are properly optimized.

\section{CONCLUSiON}

A novel hybrid IRS and FD relay network was proposed to enhance the quality of data transmission over wireless channels. A change-of-variables with SDR approach was utilized to tackle the non-convexity of the objective function and optimization constraints, and to maximize the minimum rate of the two hops of the proposed network. Our results

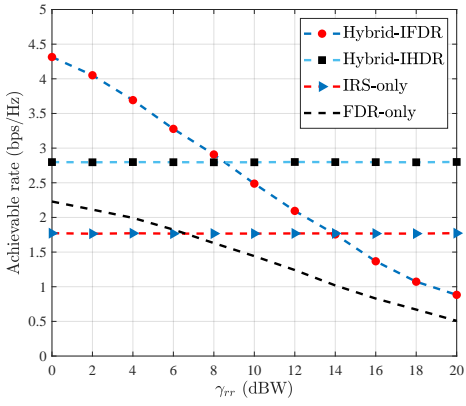

Fig. 5: Achievable rate vs $\gamma_{\mathrm{rr}}$ for different schemes when $N=$ 32 , and $\mathrm{SNR}=50 \mathrm{~dB}$.

demonstrated the significant performance gain of the proposed hybrid network compared to both the hybrid IRS with HD-DF relay and the IRS-only schemes, given that the residual selfinterference is sufficiently suppressed at the relay.

\section{REFERENCES}

[1] Q. Wu and R. Zhang, "Towards smart and reconfigurable environment: Intelligent reflecting surface aided wireless network," IEEE Commun. Mag., vol. 58, pp. 106-112, Jan. 2020.

[2] C. Huang, S. Hu, G. C. Alexandropoulos, A. Zappone, C. Yuen, R. Zhang, M. Di Renzo, and M. Debbah, "Holographic MIMO surfaces for 6G wireless networks: Opportunities, challenges, and trends," [Online]. Available: https://arxiv.org/abs/1911.12296, 2019.

[3] X. Tan, Z. Sun, J. M. Jornet, and D. Pados, "Increasing indoor spectrum sharing capacity using smart reflect-array," in IEEE Inter. Conf. Commun. (ICC), Kuala Lumpur, Malaysia, May 2016, pp. 1-6.

[4] C. Huang, R. Mo, C. Yuen et al., "Reconfigurable intelligent surface assisted multiuser MISO systems exploiting deep reinforcement learning," IEEE J. Sel. Areas Commun., to appear, 2020.

[5] Q. Wu and R. Zhang, "Beamforming optimization for wireless network aided by intelligent reflecting surface with discrete phase shifts," IEEE Trans. Commun., To appear.

[6] — , "Intelligent reflecting surface enhanced wireless network via joint active and passive beamforming," IEEE Trans. Wireless Commun., vol. 18 , no. 11, pp. 5394-5409, Nov. 2019.

[7] C. Huang, A. Zappone, G. C. Alexandropoulos, M. Debbah, and C. Yuen, "Reconfigurable intelligent surfaces for energy efficiency in wireless communication," IEEE Trans. Wireless Commun., vol. 18, no. 8, pp. 4157-4170, Aug. 2019.

[8] E. Björnson, Ö. Özdogan, and E. G. Larsson, "Intelligent reflecting surface vs. decode-and-forward: How large surfaces are needed to beat relaying?" IEEE Wireless Commun. Lett., vol. 9, no. 2, pp. 244-248, Feb. 2020.

[9] K. Ntontin, M. Di Renzo, J. Song, F. Lazarakis, J. de Rosny, D.-T. Phan-Huy, O. Simeone, R. Zhang, M. Debbah, G. Lerosey et al., "Reconfigurable intelligent surfaces vs. relaying: Differences, similarities, and performance comparison," [Online]. Available: https://arxiv.org/abs/1908.08747, 2019.

[10] Z. Abdullah, G. Chen, S. Lambotharan, and J. A. Chambers, "A hybrid relay and intelligent reflecting surface network and its ergodic performance analysis," IEEE Wireless Commun. Lett., To appear.

[11] X. Ying, U. Demirhan, and A. Alkhateeb, "Relay aided intelligent reconfigurable surfaces: Achieving the potential without so many antennas," [Online]. Available: https://arxiv.org/abs/2006.06644, 2020.

[12] P. Zhao, M. Zhang, H. Yu, H. Luo, and W. Chen, "Robust beamforming design for sum secrecy rate optimization in MU-MISO networks," IEEE Trans. Inf. Forensics Security, vol. 10, no. 9, pp. 1812-1823, Sep. 2015.

[13] Q. Wu and R. Zhang, "Intelligent reflecting surface enhanced wireless network: Joint active and passive beamforming design," in IEEE Global Commun. Conf. (GLOBECOM), Abu Dhabi, UAE. Dec. 2018, pp. 1-6.

[14] S. Zhang and Y. Huang, "Complex quadratic optimization and semidefinite programming," SIAM J. Optim., vol. 16, no. 3, pp. 871-890, 2006. 